\title{
Substantial increase in listeriosis, Denmark 2009
}

A Kvistholm Jensen (AKV@ssi.dk) ${ }^{1}$, S Ethelberg ${ }^{1,2}$, B Smith ${ }^{1}$, E Møller Nielsen ${ }^{1}$, J Larsson ${ }^{1}$, K Mølbak $^{2}$, J J Christensen ${ }^{1}$, M Kemp $^{1}$

1. Department of Microbiological Surveillance and Research, Statens Serum Institut, Copenhagen, Denmark

2. Department of Epidemiology, Statens Serum Institut, Copenhagen, Denmark

Citation style for this article:

Citation style for this article: Kvistholm Jensen A, Ethelberg S, Smith B, Møller Nielsen E, Larsson J, Mølbak K, Christensen JJ, Kemp M. Substantial increase in listeriosis, Denmark 2009. Euro Surveill. 2010;15(12):pii=19522. Available online: http://www.eurosurveillance.org/ViewArticle.aspx?Articleld=19522

This article has been published on 25 March 2010

In 2009, 97 cases of listeriosis were reported in Denmark ( 1.8 per 100,000$)$, a significant rise over the previous year. The increase was seen both in cases of bacteraemia and meningitis and affected mainly people aged 70 years and older. A foodborne outbreak of eight cases was identified by pulsed-field gel electrophoresis typing. No explanation has so far been found for the marked increase in incidence. An increasing trend has been observed since 2003 and possible explanations are discussed.

\section{Introduction}

Invasive listeriosis is a serious foodborne infection caused by Listeria monocytogenes. The three major clinical presentations of listeriosis are sepsis, meningitis and materno-fetal infection. In a review of maternofetal infections in Denmark 12 of 36 cases resulted in stillbirth and/or abortion [1]. In recent years the annual number of reported listeriosis cases has increased in several European countries including Denmark [2-4]. However, case definitions, diagnostic practices and surveillance systems differ across European countries, factors that must be taken into consideration when interpreting these data. In 2009, Denmark saw a further increase in the incidence of listeriosis, reaching 1.8 per 100,000 inhabitants. To our knowledge this is the highest incidence reported in a European country in recent years. In this paper, data from the Danish surveillance system are presented and discussed.

\section{Materials and Methods}

Culture-confirmed cases of listeriosis are notifiable by Danish diagnostic laboratories to Statens Serum Institut (SSI). Information on age, sex, isolation site, collection date of the specimen and hospital department from which the specimen is sent is compulsory. The laboratories also refer the isolated bacterium for typing. Data on the clinical presentation (septicaemia, meningitis or other), predisposing factors, antibiotic therapy, medicine and the general condition of the patient are reported on a voluntary basis. The case definition for listeriosis used in Denmark is according to the case definition by the European Commission [5]. Cases are divided into groups of sepsis, meningitis, materno-fetal infection or other, according to the site of isolation of $L$. monocytogenes and/or clinical presentation reported in the patients' charts. Hence a patient with a clinical diagnosis of meningitis may be counted as such even if $L$. monocytogenes was not isolated from cerebrospinal fluid but from another body fluid. Materno-fetal cases include pregnancy-associated cases and listeriosis in newborns in the first month of life. A materno-fetal infection counts as one case and is reported on the mother.

For the present report, mortality information was obtained from the Danish Civil Registry System (CPRregistret). To estimate the case fatality rate, death within 30 days of sample date was arbitrarily defined as death related to listeriosis. $L$. monocytogenes isolates from human cases are routinely typed by pulsed-field gel electroforesis (PFGE) to detect clusters as a means to survey for outbreaks. PFGE is performed according to the PulseNet method using the two enzymes Ascl and Apal [6]. The number of cases in 2009 was compared to the number of cases in 2008. A $P$ value was calculated using a likelihood ratio test, under the assumption that the annual number of cases follows a Poisson distribution.

\section{Results}

In 2009, 97 cases of listeriosis (1.8 per 100,000 population) were reported in Denmark, compared to 57 in $2008(P=0.0014)$. Fifty cases were in females; three were materno-fetal infections. In the period from 1989 to 2008 , the annual incidence varied between 0.4 and 1.1 per 100,000 (Figure 1).

The incidences in the age groups were $0.4,3.7,7.3,12.1$ and 22.0 per 100,000 for the age groups 0-59, 60-69, 70-79, 80-89 and 90+ years, respectively (Figure 2).

A review of the patients' clinical information did not reveal predisposing factors to listeriosis other than those already known (malignancies, diabetes mellitus, old age, pregnancy, immunosuppressing diseases and treatment) [7]. As not all clinical data from previous years are available as yet, a comparison with previous years was not possible for this analysis, however, the available data do not exclude that there may have been an increase in a single patient group. The distribution of clinical manifestations is depicted in Figure 3. The 
sepsis to meningitis ratio remained at approximately five, largely unchanged compared with previous years. The incidence of materno-fetal infection was 4.8 per 100,000 live births. This was not higher than seen previously [1].

The case fatality rate was $28 \%$ in 2009 , which was similar to previous years [7]. PFGE typing revealed 51 PFGE types among the 97 isolates from 2009. The most

\section{FIGURE 1}

Annual incidence of listeriosis per 100,000 inhabitants, Denmark, 1989-2009

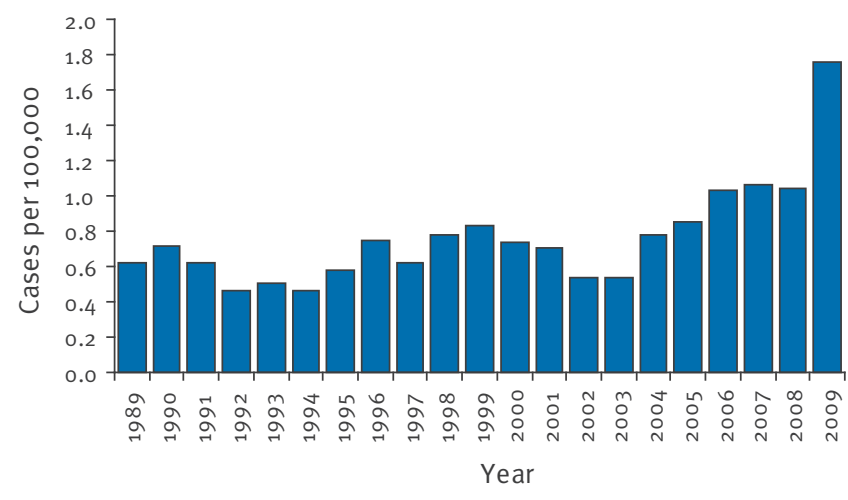

\section{FIGURE 2}

Annual incidence of listeriosis per 100,000 inhabitants by age group, Denmark, 2003-2009

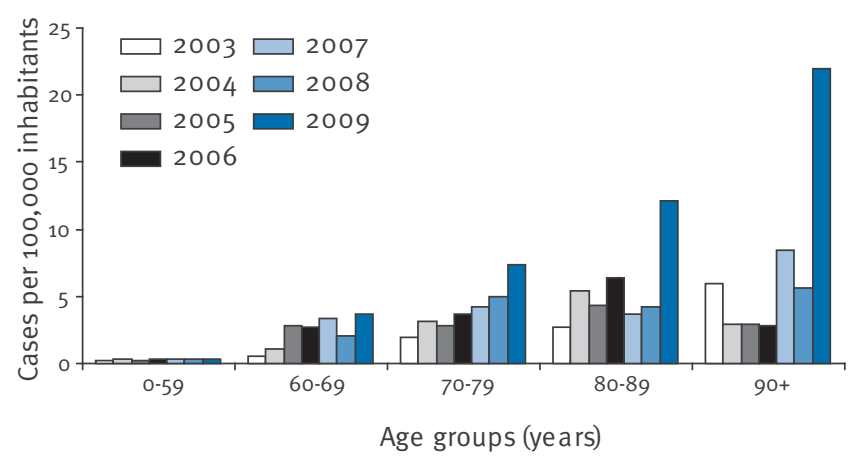

\section{FIGURE 3}

Distribution of clinical manifestations in reported listeriosis cases, Denmark, 2003-2009

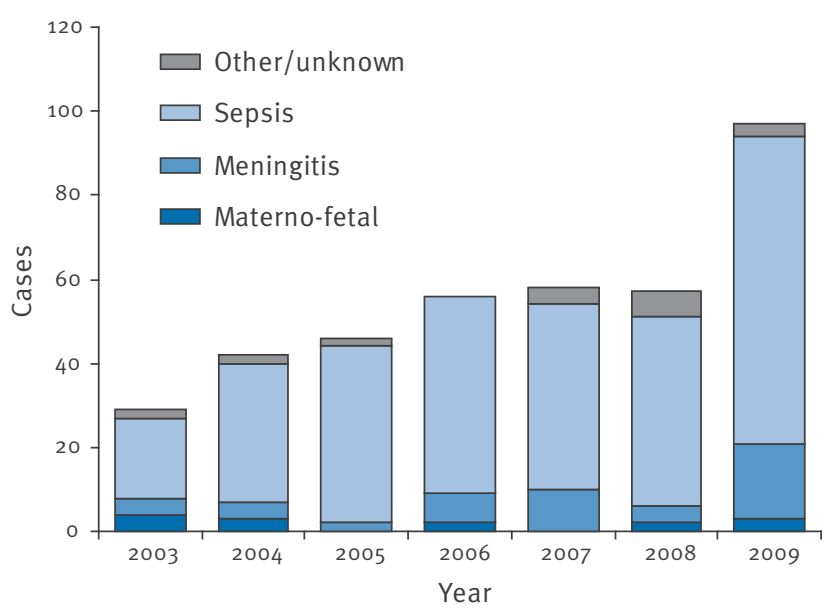

common type, type 42-40 (first profile in Figure 4) with 16 isolates scattered throughout the year, had also been commonly seen in previous years, representing $21 \%$ of the isolates in 2006 to 2009.

In the spring of 2009, a verified outbreak included eight cases [8]. The involved PFGE type (type 43-71; Figure 4) had not previously been seen in Denmark. In the autumn of 2009, a cluster of seven cases was identified by PFGE, but could not be confirmed as an outbreak. The PFGE type of this cluster (type 23-03; Figure 4 ) is a fairly common type represented by between two and five cases per year in previous years.

\section{Discussion}

There has been a general increasing trend in the number of listeriosis cases in Denmark since 2003 and an additional marked increase from 2008 to 2009. The increase in listeriosis incidence in 2009 was seen particularly in the group of patients over 70 years of age. There is no single explanation for this dramatic increase. PFGE typing showed a high diversity of isolates in 2009 as well as in previous years, and the rise cannot be explained by a higher number of cases with any specific $L$. monocytogenes type. Even disregarding the 15 patients from the two clusters, the incidence is still very high (1.5 per 100,000). It is difficult to explain the steep increase from 2008 to 2009. However, several possible explanations for the general increase are conceivable; examples of such are listed in the Table.

There are indications that the consumption of readyto-eat (RTE) products has increased. Consumer surveys from Statistics Denmark, comparing the period from 2005 to 2007 with the period from 2003 to 2005 indicates that expenditures for RTE products have increased by $87 \%$ for RTE meat products and by $34 \%$ for RTE fish products among individuals older than 60 years and living alone [9]. It will be valuable to obtain data on the frequency of contamination of RTE products with L. monocytogenes from the survey recently launched by the European Food Safety Authority [10], which should aid in assessing whether consumption of these products could represent a risk factor for acquiring listeriosis.

Susceptibility in the population may increase if the group of persons with predisposing factors, including immunosuppressive conditions and high age, grows [11], for instance due to better medical treatment and survival of seriously ill people such as cancer patients. As seen in Figure 2, the incidence has risen substantially within the group of over 70-year-olds, so demographic changes alone do not explain the increase. Because an increase is seen in sepsis and in meningitis cases (Figure 3), it seems unlikely that it can be explained by improved routines in taking blood cultures. Nor have, to our knowledge, healthcare practices and reporting procedures changed in recent years. 
Another hypothesis to explain the increase in incidence would be that changes in empirical antibiotic therapy of patients presenting with sepsis could contribute to higher detection rates. While Danish recommendations formerly advised a combination of a penicillin and an aminoglycoside for the treatment of sepsis, the practice has now changed in many places in favour of cephalosporines as first-choice antibiotics. Cephalosporines, to which L. monocytogenes is resistant, are now the most used group of antibiotics in hospitals [12]. If samples for culture are taken after initiation of systemic antibiotic treatment, this could result in fewer falsenegative samples. However, the change in treatment has come over several years and while it could have contributed to the gradual increase seen over several years, it seems unlikely that this alone would explain an increase from one year to another.

Unexplained year-to-year fluctuations in the incidence of listeriosis have been reported from several countries [2-4], and could be due to random variations in incidence. In 2010, seven cases of listeriosis have been reported in Denmark as of 1 March. Over the last 17 years, the number of cases in the two first months of the year has varied between two and 13, and it is still too early to predict a trend for 2010 . The human, food and environmental sectors involved in listeriosis surveillance in Denmark are currently working together to gather all relevant information about the situation in order to find possible explanations and strategies for future intervention and prevention. Further investigation into typing of isolates and into consumption and handling of foods in the at-risk groups are among the possibilities being considered. Hopefully the results of these investigations will give indications as to possible public health interventions. Communications from other European countries on the situation and suggestions to explain the rise in cases seen in several countries will be of high value.

\section{FIGURE 4}

Representatives of the most common PFGE profiles in listeria isolates in Denmark in 2006-2009 (listed according to prevalence)

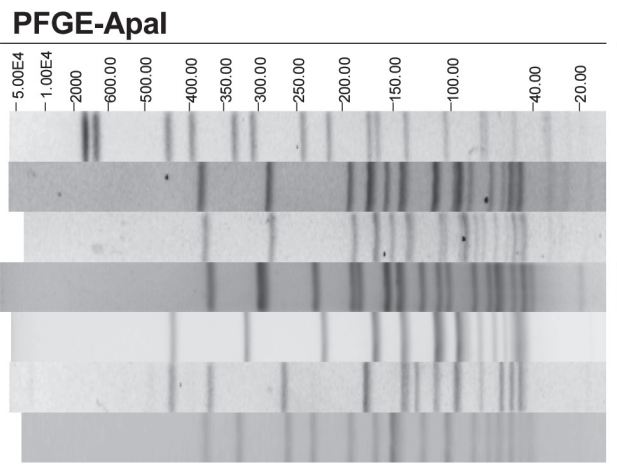

\section{PFGE-Ascl}

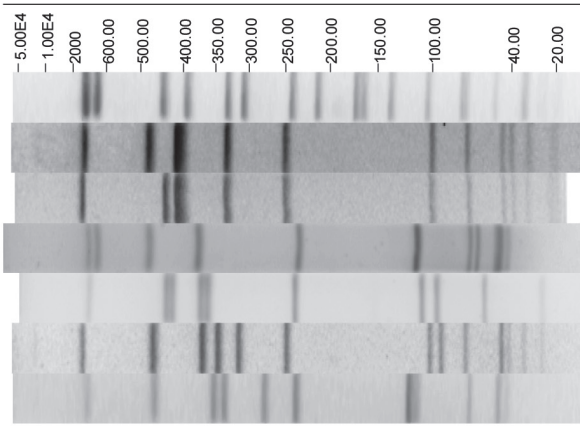

PFGE-Apal-pattern PFGE-Ascl-pattern marker

GX6A12.0042.DK

GX6A12.0048.DK

GX6A12.0023.DK

GX6A12.0063.DK

GX6A12.0043.DK

GX6A12.0092.DK

marker

GX6A16.0040.DK

GX6A16.0038.DK

GX6A16.0003.DK

GX6A16.0043.DK

GX6A16.0071.DK

GX6A16.0067.DK

PFGE: pulsed-field gel electrophoresis.

The clusters in spring and autumn had the types 43-71 and 92-67, respectively. PulseNet nomenclature for type 43-71 is Apal-pattern GXBA12.0043.DK, Ascl-pattern GXBA16.0071.DK.

\section{TABLE}

Potential explanations for the observed increase in the number of Listeria monocytogenes infections in Denmark

\begin{tabular}{|l|l|}
\hline Overall explanation & Examples \\
\hline Increased exposure & Increased consumption of ready-to-eat products \\
\hline & Higher levels of contamination in specific products \\
\hline Demographic changes & Suboptimal food storage conditions by the consumers \\
\hline & More elderly in the population \\
\hline & More persons alive with illness predisposing to listeriosis \\
\hline Outbreaks & More people taking medicine predisposing to listeriosis \\
\hline Changes in strains & People infected with specific bacterial strains from the same food source \\
\hline Surveillance artefacts & Increased virulence of $L$ monocytogenes isolates from patients \\
\hline & Increased number of blood cultures taken \\
\hline & Increased reporting \\
\hline & Better diagnostic methods \\
\hline & Changes in the empirical use of antibiotics in the hospital systems \\
\hline
\end{tabular}




\section{References}

1. Smith B, Kemp M, Ethelberg S, Schiellerup P, Bruun BG, Gerner-Smidt P, et al. Listeria monocytogenes: maternalfoetal infections in Denmark 1994-2005. Scand J Infect Dis. 2009;41(1):21-5.

2. Allerberger F, Wagner M. Listeriosis: a resurgent foodborne infection Clin Microbiol Infect. 2010;16(1):16-23.

3. Goulet V, Hedberg C, Le Monnier A., de Valk H. Increasing incidence of listeriosis in France and other European countries. Emerg Infect Dis. 2008;14(5):734-40.

4. Denny J, McLauchlin J. Human Listeria monocytogenes infections in Europe--an opportunity for improved European surveillance. Euro Surveill. 2008;13(13). pii =8082. Available from: http://www.eurosurveillance.org/ViewArticle. aspx?Articleld $=8082$

5. European Commission. 2008/351/EC: Commission Decision of 28 April 2008 amending Decision 2002/253/EC laying down case definitions for reporting communicable diseases to the Community network under Decision No 2119/98/EC of the European Parliament and of the Council" (notified under document number C(2008) 1589) (Text with EEA relevance) (2008/426/EC). Available from: http://eur-lex.europa.eu/ LexUriServ/LexUriServ.do?uri=0J:L:2008:117:0040:0041:EN:P DF

6. Graves LM, Swaminathan B. PulseNet standardized protocol for subtyping Listeria monocytogenes by macrorestriction and pulsed-field gel electrophoresis. Int J Food Microbiol. 2001;65(1-2):55-62.

7. Gerner-Smidt P, Ethelberg S, Schiellerup P, Christensen JJ, Engberg J, Fussing V, et al. Invasive listeriosis in Denmark 1994-2003: a review of 299 cases with special emphasis on risk factors for mortality. Clin Microbiol Infect. 2005;11(8):618-24.

8. Smith B, Larsson JT, Lisby M, Muller L, Madsen SB, Engberg J, et al. Outbreak of Listeria monocytogenes caused by Beef meat from a Meals-on-Wheel's delivery, Denmark 2009. Clin Microbiol Infect. 2010; Feb 24. [Epub ahead of print]

9. Household yearly consumption by type of consumption and group of households. FU 5 . Copenhagen: Statistics Denmark. [Internet]. Available from: http://www. statistikbanken.dk/statbank5a/SelectVarVal/Define. asp? Maintable $=F U_{5} \&$ PLanguage $=1$

10. European Food Safety Authority. Report of Task Force on Zoonoses Data Collection on proposed technical specifications for a survey on Listeria monocytogenes in selected categories of ready-to-eat food at retail in the EU, The EFSA Journal, (2009), 2616, 1- 66. Available from: http://www.efsa.europa. eu/en/scdocs/scdoc/30or.htm

11. Gillespie IA, McLauchlin J, Grant KA, Little CL, Mithani V, Penman C, et al. Changing pattern of human listeriosis, England and Wales, 2001-2004. Emerg Infect Dis. 2006;12(9):1361-6.

12. Danish Integrated Antimicrobial resistance Monitoring and Research Programme (DANMAP). DANMAP 2008 - Use of antimicrobial agents and occurrence of antimicrobial resistance in bacteria from food animals, foods and humans in Denmark. National Food Institute; Technical University of Denmark. Soborg; 2009. Available from: http://www.danmap. org/pdfFiles/Danmap_2008.pdf 